\title{
ON THE COMPLEXITY OF DETECTING POSITIVE EIGENVECTORS OF NONLINEAR CONE MAPS
}

\author{
BAS LEMMENS AND LEWIS WHITE
}

\begin{abstract}
In recent work with Lins and Nussbaum the first author gave an algorithm that can detect the existence of a positive eigenvector for orderpreserving homogeneous maps on the standard positive cone. The main goal of this paper is to determine the minimum number of iterations this algorithm requires. It is known that this number is equal to the illumination number of the unit ball, $B_{\mathrm{v}}$, of the variation norm, $\|x\|_{\mathrm{v}}:=\max _{i} x_{i}-\min _{i} x_{i}$ on $V_{0}:=\left\{x \in \mathbb{R}^{n}: x_{n}=0\right\}$. In this paper we will show that the illumination number of $B_{\mathrm{V}}$ is equal to $\left(\begin{array}{c}n \\ \left\lceil\frac{n}{2}\right\rceil\end{array}\right)$, and hence provide a sharp lower bound for the running time of the algorithm.
\end{abstract}

\section{INTRODUCTION}

The classical Perron-Frobenius theory concerns the spectral properties of square nonnegative matrices. In recent decades this theory has been extended to a variety of nonlinear maps that preserve a partial ordering induced by a cone, see [8] and the references therein for an up-to-date account.

Of particular interest are order-preserving homogeneous maps $f: \mathbb{R}_{\geq 0}^{n} \rightarrow \mathbb{R}_{\geq 0}^{n}$, where

$$
\mathbb{R}_{\geq 0}^{n}:=\left\{x \in \mathbb{R}^{n}: x_{i} \geq 0 \text { for all } i=1, \ldots, n\right\}
$$

is the standard positive cone. Recall that $f: \mathbb{R}_{\geq 0}^{n} \rightarrow \mathbb{R}_{\geq 0}^{n}$ is order-preserving if $f(x) \leq f(y)$ whenever $x \leq y$ and $x, y \in \mathbb{R}_{\geq 0}^{n}$. Here $w \leq z$ if $z-w \in \mathbb{R}_{\geq 0}^{n}$. Furthermore, $f$ is said to be homogeneous if $f(\lambda x)=\lambda f(x)$ for all $\lambda \geq 0$ and $x \in \mathbb{R}_{\geq 0}^{n}$. Such maps arise in mathematical biology $[10,13]$ and in optimal control and game theory $[1,12]$.

It is known [8, Corollary 5.4.2] that if $f: \mathbb{R}_{\geq 0}^{n} \rightarrow \mathbb{R}_{\geq 0}^{n}$ is a continuous, orderpreserving, homogeneous map, then there exists $v \in \mathbb{R}_{\geq 0}^{n}$ such that

$$
f(v)=r(f) v,
$$

where

$$
r(f):=\lim _{k \rightarrow \infty}\left\|f^{k}\right\|_{\mathbb{R}_{\geq 0}^{n}}^{1 / k}
$$

is the cone spectral radius of $f$ and

$$
\|g\|_{\mathbb{R}_{\geq 0}^{n}}:=\sup \left\{\|g(x)\|: x \in \mathbb{R}_{\geq 0}^{n} \text { and }\|x\| \leq 1\right\} .
$$

2010 Mathematics Subject Classification. Primary 47H07, 47H09;; Secondary 37C25.

Key words and phrases. Nonlinear maps on cones, positive eigenvectors, illumination problem, Hilbert's metric.

The second author was supported by a London Mathematical Society "Undergraduate Research Bursary" and the School of Mathematics, Statistics and Actuarial Science at the University of Kent. 
Thus, as in the case of nonnegative matrices, continuous order-preserving homogeneous maps on $\mathbb{R}_{\geq 0}^{n}$ have an eigenvector in the cone corresponding to the spectral radius.

In many applications it is important to know if the map has a positive eigenvector, i.e., an eigenvector that lies in the interior, $\mathbb{R}_{>0}^{n}:=\left\{x \in \mathbb{R}_{\geq 0}^{n}: x_{i}>0\right.$ for $i=$ $1, \ldots, n\}$, of $\mathbb{R}_{\geq 0}^{n}$. This appears to be a much more subtle problem. There exists a variety of sufficient conditions in the literature, see [4], [5], [8, Chapter 6], and [9]. Recently, Lemmens, Lins and Nussbaum [7, Section 5] gave an algorithm that can confirm the existence of a positive eigenvector for continuous, order-preserving, homogeneous maps $f: \mathbb{R}_{\geq 0}^{n} \rightarrow \mathbb{R}_{\geq 0}^{n}$. The main goal of this paper is to determine the minimum number of iterations this algorithm needs to perform.

\section{Preliminaries}

Before we state the main result we give some more background. First let us introduce some notation. Given a set $S$ in a finite dimensional vector space $V$ we write $S^{\circ}$ to denote the interior of $S$, and $\partial S$ to denote the boundary of $S$ with respect to the norm topology on $V$.

It is known that if $f: \mathbb{R}_{\geq 0}^{n} \rightarrow \mathbb{R}_{\geq 0}^{n}$ is an order-preserving homogeneous map and there exists $z \in \mathbb{R}_{>0}^{n}$ such that $f(\bar{z}) \in \partial \mathbb{R}_{\geq 0}^{n}$, then $f\left(\mathbb{R}_{>0}^{n}\right) \subset \partial \mathbb{R}_{\geq 0}^{n}$, see $[8$, Lemma 1.2.2]. Thus to analyse the existence of a positive eigenvector one may as well consider order-preserving homogeneous maps $f: \mathbb{R}_{>0}^{n} \rightarrow \mathbb{R}_{>0}^{n}$. Moreover, on $\mathbb{R}_{>0}^{n}$ we have Hilbert's metric, $d_{H}$, which is given by

$$
d_{H}(x, y):=\log \left(\max _{i} \frac{x_{i}}{y_{i}}\right)-\log \left(\min _{i} \frac{x_{i}}{y_{i}}\right) \quad \text { for } x, y \in \mathbb{R}_{>0}^{n} .
$$

Note that $d_{H}$ is not a genuine metric, as $d_{H}(\lambda x, \mu x)=0$ for all $x \in \mathbb{R}_{>0}^{n}$ and $\lambda, \mu>0$. In fact, $d_{H}(x, y)=0$ if and only if $x=\lambda y$ for some $\lambda>0$. However, $d_{H}$ is a metric on the set of rays in $\mathbb{R}_{>0}^{n}$.

If $f: \mathbb{R}_{>0}^{n} \rightarrow \mathbb{R}_{>0}^{n}$ is order-preserving and homogeneous, then $f$ is nonexpansive under $d_{H}$, i.e.,

$$
d_{H}(f(x), f(y)) \leq d_{H}(x, y) \quad \text { for all } x, y \in \mathbb{R}_{>0}^{n},
$$

see for example [8, Proposition 2.1.1]. In particular, order-preserving homogeneous maps $f: \mathbb{R}_{>0}^{n} \rightarrow \mathbb{R}_{>0}^{n}$ are continuous on $\mathbb{R}_{>0}^{n}$. Moreover, if $x$ and $y$ are eigenvectors of $f: \mathbb{R}_{>0}^{n} \rightarrow \mathbb{R}_{>0}^{n}$ with $f(x)=\lambda x$ and $f(y)=\mu y$, then $\lambda=\mu$, see [8, Corollary $5.2 .2]$.

In [7, Theorem 5.1] the following necessary and sufficient conditions were obtained for an order-preserving homogeneous map $f: \mathbb{R}_{>0}^{n} \rightarrow \mathbb{R}_{>0}^{n}$ to have a nonempty set of eigenvectors, $\mathrm{E}(f):=\left\{x \in \mathbb{R}_{>0}^{n}: x\right.$ eigenvector of $\left.f\right\}$, which is bounded under Hilbert's metric.

Theorem 2.1. If $f: \mathbb{R}_{>0}^{n} \rightarrow \mathbb{R}_{>0}^{n}$ is an order-preserving homogeneous map, then $\mathrm{E}(f)$ is nonempty and bounded under $d_{H}$ if and only if for each nonempty proper subset $J$ of $\{1, \ldots, n\}$ there exists $x^{J} \in \mathbb{R}_{>0}^{n}$ such that

$$
\max _{j \in J} \frac{f\left(x^{J}\right)_{j}}{x_{j}^{J}}<\min _{j \in J^{c}} \frac{f\left(x^{J}\right)_{j}}{x_{j}^{J}} .
$$

Note that the assertion is trivial in case $n=1$, as each order-preserving homogeneous map $f: \mathbb{R}_{>0} \rightarrow \mathbb{R}_{>0}$ has a nonempty bounded set of eigenvectors. In 
case $n \geq 2$ Theorem 2.1 yields the following simple algorithm for detecting positive eigenvectors:

Algorithm 2.2. Let $f: \mathbb{R}_{>0}^{n} \rightarrow \mathbb{R}_{>0}^{n}$ be an order-preserving homogeneous map. Repeat the following steps until every nonempty proper subset $J$ of $\{1, \ldots, n\}$ has been recorded.

Step 1: Randomly select $x$, with $0<x_{j}<1$ for all $j$, and compute $f(x)_{j} / x_{j}$ for all $j \in\{1, \ldots, n\}$.

Step 2: Record all nonempty proper subsets $J \subset\{1, \ldots, n\}$ such that inequality (2.1) holds.

So, if this algorithm halts, then $f$ has an eigenvector in $\mathbb{R}_{>0}^{n}$. On the other hand, if $\mathrm{E}(f)$ is empty or unbounded under $d_{H}$, then the algorithm will never halt. Note that a randomly chosen $x$ in Step 1 can eliminate multiple subsets $J$ in Step 2 . So, it is natural to ask for the least number of vectors required to fulfill the $2^{n}-2$ inequalities in (2.1). This number corresponds to the minimum number of times the algorithm has to perform Steps 1 and 2. In this paper we will show that one needs at least

$$
\left(\begin{array}{c}
n \\
\lceil n / 2\rceil
\end{array}\right)
$$

vectors and this lower bound is sharp. Here $\lceil a\rceil$ is the smallest integer $n \geq a$. Likewise we shall write $\lfloor a\rfloor$ to denote the largest integer $n \leq a$.

\section{Connection With the illumination Number}

Recall that given a compact convex set $C$ with nonempty interior in $V$, a vector $v \in V$ illuminates $z \in \partial C$ if $z+\lambda v \in C^{\circ}$ for all $\lambda>0$ sufficiently small. A set $S$ is said to Illuminate $C$ if for each $z \in \partial C$ there exists $v \in S$ such that $v$ illuminates $z$. The minimal size of illuminating set for $C$ is called the illumination number of $C$ and is denoted $i(C)$. There is a long-standing open conjecture which asserts that $i(C) \leq 2^{n}$ for every compact convex body in an $n$-dimensional vector space, see $[2$, Chapter VI] for further details. It is easy to show, see for example [7, Lemma 4.1], that if $S$ illuminates every extreme point of $C$, then $S$ illuminates $C$.

To proceed we need to discuss the connection between illumination numbers and Theorem 2.1. Firstly, we note that if we let $\Sigma_{0}:=\left\{x \in \mathbb{R}_{>0}^{n}: x_{n}=1\right\}$, then $\left(\Sigma_{0}, d_{H}\right)$ is a metric space. Given an order-preserving homogeneous map $f: \mathbb{R}_{>0}^{n} \rightarrow \mathbb{R}_{>0}^{n}$ we can consider the normalised map $g_{f}: \Sigma_{0} \rightarrow \Sigma_{0}$ given by

$$
g_{f}(x):=\frac{f(x)}{f(x)_{n}} \quad \text { for } x \in \Sigma_{0} .
$$

The map $g_{f}$ is nonexpansive under $d_{H}$ on $\Sigma_{0}$. Moreover, $x \in \Sigma_{0}$ is a fixed point of $g_{f}$ if and only if $x$ is an eigenvector of $f$. Thus, if we let $\operatorname{Fix}\left(g_{f}\right):=\{x \in$ $\left.\Sigma_{0}: g_{f}(x)=x\right\}$, then $\operatorname{Fix}\left(g_{f}\right)$ is nonempty and bounded in $\left(\Sigma_{0}, d_{H}\right)$ if and only if $\mathrm{E}(f)$ is nonempty and bounded in $\left(\mathbb{R}_{>0}^{n}, d_{H}\right)$.

It not hard to verify that the map $\log : \Sigma_{0} \rightarrow V_{0}$ given by

$$
\log (x):=\left(\log x_{1}, \ldots, \log x_{n}\right) \quad \text { for } x=\left(x_{1}, \ldots, x_{n}\right) \in \Sigma_{0}
$$

is an isometry from $\left(\Sigma_{0}, d_{H}\right)$ onto $\left(V_{0},\|\cdot\|_{\mathrm{v}}\right)$, where $V_{0}:=\left\{x \in \mathbb{R}^{n}: x_{n}=0\right\}$ and

$$
\|x\|_{\mathrm{v}}:=\max _{i} x_{i}-\min _{i} x_{i}
$$

is the variation norm. 
It follows that the map $h: V_{0} \rightarrow V_{0}$ satisfying $h \circ \log =\log \circ g_{f}$ is nonexpansive under the variation norm, and $\operatorname{Fix}(h)$ is nonempty and bounded in $\left(V_{0},\|\cdot\|_{\mathrm{v}}\right)$ if and only if $\operatorname{Fix}\left(g_{f}\right)$ is nonempty and bounded in $\left(\Sigma_{0}, d_{H}\right)$.

In $[7$, Theorem 3.4] the following result concerning fixed point sets of nonexpansive maps on finite dimensional normed spaces was proved.

Theorem 3.1. If $h: V \rightarrow V$ is a nonexpansive map on a finite dimensional normed space $V$, then $\operatorname{Fix}(h)$ is nonempty and bounded if and only if there exist $w^{1}, \ldots, w^{m} \in V$ such that $\left\{f\left(w^{i}\right)-w^{i}: i=1, \ldots, m\right\}$ illuminates the unit ball of $V$.

For $n \geq 2$, the unit ball $B_{\mathrm{v}}$ of $\left(V_{0},\|\cdot\|_{\mathrm{v}}\right)$ has $2^{n}-2$ extreme points, which are given by

$$
\operatorname{ext}\left(B_{\mathrm{v}}\right):=\left\{v_{+}^{I}: \emptyset \neq I \subseteq\{1, \ldots, n-1\}\right\} \cup\left\{v_{-}^{I}: \emptyset \neq I \subseteq\{1, \ldots, n-1\}\right\},
$$

where $\left(v_{+}^{I}\right)_{i}=1$ if $i \in I$ and 0 otherwise, and $\left(v_{-}^{I}\right)_{i}=-1$ if $i \in I$ and 0 otherwise. See $[11, \S 2]$ for details.

In [7] the equivalence in Theorem 2.1 was obtained by using Theorem 3.1 and showing that there exists $x^{1}, \ldots, x^{m} \in \mathbb{R}_{>0}^{n}$ that fulfill the $2^{n}-2$ inequalities in (2.1) if and only if there exist $y^{1}, \ldots, y^{m} \in V_{0}$ that illuminate the $2^{n}-2$ extreme points of the unit ball $B_{\mathrm{v}}$. Thus, $i\left(B_{\mathrm{v}}\right)$ provides a sharp lower bound for the number of times one needs to repeat Steps 1 and 2 in Algorithm 2.2. In the next section we will show the following result concerning $i\left(B_{\mathrm{v}}\right)$.

Theorem 3.2. If $B_{\mathrm{v}}$ is the unit ball of $\left(V_{0},\|\cdot\|_{\mathrm{v}}\right)$ and $n \geq 2$, then

$$
i\left(B_{\mathrm{v}}\right)=\left(\begin{array}{c}
n \\
\lceil n / 2\rceil
\end{array}\right) .
$$

\section{Proof of Theorem 3.2}

Note that the map $\left(x_{1}, \ldots, x_{n}\right) \in V_{0} \mapsto\left(x_{1}, \ldots, x_{n-1}\right) \in \mathbb{R}^{n-1}$ is an isometry from $\left(V_{0},\|\cdot\|_{\mathrm{v}}\right)$ onto $\left(\mathbb{R}^{n-1},\|\cdot\|_{H}\right)$, where

$$
\|x\|_{H}:=\left(\max _{i} x_{i}\right) \vee 0-\left(\min _{i} x_{i}\right) \wedge 0 .
$$

Here $a \wedge b:=\min (a, b)$ and $a \vee b:=\max (a, b)$. Note also that if $B_{H}$ is the unit ball in $\left(\mathbb{R}^{n-1},\|\cdot\|_{H}\right)$, then

$$
\operatorname{ext}\left(B_{H}\right)=\left(\{0,1\}^{n-1} \cup\{0,-1\}^{n-1}\right) \backslash\{(0, \ldots, 0)\}
$$

and

$$
i\left(B_{H}\right)=i\left(B_{\mathrm{v}}\right) .
$$

For notational simplicity we will work with $B_{H}$ instead of $B_{\mathrm{v}}$.

The following two subsets,

$$
E_{+}:=\{0,1\}^{n-1} \backslash\{(0, \ldots, 0)\} \quad \text { and } \quad E_{-}:=\{0,-1\}^{n-1} \backslash\{(0, \ldots, 0)\},
$$

of $\operatorname{ext}\left(B_{H}\right)$ play a key role in the argument. On $\operatorname{ext}\left(B_{H}\right)$ we have the usual partial ordering $x \leq y$ if $y-x \in \mathbb{R}_{>0}^{n-1}$, which gives rise to two finite partially ordered sets $\left(E_{+}, \leq\right)$and $\left(E_{-}, \leq\right)$.

Recall that subset $\mathcal{A}$ of a partially ordered set $(P, \preceq)$ is called an antichain if $x, y \in \mathcal{A}$ and $x \preceq y$ implies $x=y$. A chain $\mathcal{C}$ in $(P, \preceq)$ is a totally ordered subset, if for each $x, y \in \mathcal{C}$ we have that either $x \preceq y$ or $y \preceq x$. The length of a chain $\mathcal{C}$ is the number of distinct elements in $\mathcal{C}$. 
Lemma 4.1. Let $\mathcal{A}$ be an antichain in $\left(E_{+}, \leq\right)$or in $\left(E_{-}, \leq\right)$. If $x \neq y$ in $\mathcal{A}$ are illuminated by $v$ and $w$, respectively, then $v \neq w$.

Proof. Suppose that $\mathcal{A}$ is antichain in $\left(E_{+}, \leq\right)$and $x \neq y$ are in $\mathcal{A}$. Then there exist $i \neq j$ such that $0=x_{i}<y_{i}=1$ and $0=y_{j}<x_{j}=1$. Now suppose by way of contradiction that $z$ illuminates $x$ and $y$. So, $\|x+\lambda z\|_{H}<1$ and $\|y+\lambda z\|_{H}<1$ for all $\lambda>0$ sufficiently small. Suppose first that $z_{i} \leq z_{j}$. Then for $\lambda>0$ small,

$$
1+\lambda z_{j}=x_{j}+\lambda z_{j} \leq\|x+\lambda z\|_{H}<1,
$$

and hence $z_{j}<0$. So, $z_{i} \leq z_{j}<0$. But then

$$
1+\lambda\left(z_{j}-z_{i}\right)=x_{j}+\lambda z_{j}-\lambda z_{i} \leq\|x+\lambda z\|_{H}<1,
$$

which is impossible. On the other hand, if $z_{j} \leq z_{i}$, then $1+\lambda z_{i} \leq\|y+\lambda z\|_{H}<1$, so that $z_{j} \leq z_{i}<0$. But then

$$
1+\lambda\left(z_{i}-z_{j}\right)=y_{i}+\lambda z_{i}-\lambda z_{j} \leq\|y+\lambda z\|_{H}<1,
$$

which again is impossible. Thus, $z$ cannot illuminate both $x$ and $y$.

The argument for the case where $\mathcal{A}$ is antichain in $\left(E_{-}, \leq\right)$is similar.

Lemma 4.2. If $x, y \in \operatorname{ext}\left(B_{H}\right)$ are such that $x_{i}=1$ and $y_{i}=-1$ for some $i$, then one needs two distinct vectors to illuminate $x$ and $y$.

Proof. Suppose $w$ illuminates $x$ and $y$. Then $1+\lambda w_{i}=x_{i}+\lambda w_{i} \leq\|x+\lambda w\|_{H}<1$ for all $\lambda>0$ sufficiently small, and hence $w_{i}<0$. But also $1-\lambda w_{i}=-\left(y_{i}+\lambda w_{i}\right) \leq$ $\|y+\lambda w\|_{H}<1$ for all $\lambda>0$ sufficiently small. This implies that $w_{i}>0$, which is impossible. Thus, one needs at least two vectors to illuminate $x$ and $y$.

Corollary 4.3. If $B_{H}$ is the unit ball of $\left(\mathbb{R}^{n-1},\|\cdot\|_{H}\right)$ and $n \geq 2$, then

$$
i\left(B_{H}\right) \geq\left(\begin{array}{c}
n \\
\lceil n / 2\rceil
\end{array}\right) \text {. }
$$

Proof. For $1 \leq k, m \leq n-1$ define the antichians $\mathcal{A}_{+}(k):=\left\{x \in E_{+}: \sum_{i} x_{i}=k\right\}$ and $\mathcal{A}_{-}(m):=\left\{x \in E_{-}: \sum_{i} x_{i}=-m\right\}$. If $n>1$ is odd, then we can take $k:=(n-1) / 2$ and $m:=(n+1) / 2$ and conclude from Lemmas 4.1 and 4.2 that we need at least

$$
\left(\begin{array}{c}
n-1 \\
\frac{n-1}{2}
\end{array}\right)+\left(\begin{array}{c}
n-1 \\
\frac{n+1}{2}
\end{array}\right)=\left(\begin{array}{c}
n \\
\left\lceil\frac{n}{2}\right\rceil
\end{array}\right)
$$

distinct vectors to illuminate the extreme points in $\mathcal{A}_{+}(k) \cup \mathcal{A}_{-}(m)$, as for each $x \in \mathcal{A}_{+}(k)$ and $y \in \mathcal{A}_{-}(m)$ there exists an $i$ such that $x_{i}=1$ and $y_{i}=-1$.

Likewise if $n>1$ is even, we can take $k=m=\left\lceil\frac{n-1}{2}\right\rceil$, and deduce from Lemmas 4.1 and 4.2 that we need at least

$$
\left(\begin{array}{c}
n-1 \\
\left\lceil\frac{n-1}{2}\right\rceil
\end{array}\right)+\left(\begin{array}{c}
n-1 \\
\left\lceil\frac{n-1}{2}\right\rceil
\end{array}\right)=\left(\begin{array}{c}
n-1 \\
\left\lfloor\frac{n-1}{2}\right\rfloor
\end{array}\right)+\left(\begin{array}{c}
n-1 \\
\left\lceil\frac{n-1}{2}\right\rceil
\end{array}\right)=\left(\begin{array}{c}
n \\
\frac{n}{2}
\end{array}\right)
$$

distinct vectors to illuminate the extreme points in $\mathcal{A}_{+}(k) \cup \mathcal{A}_{-}(m)$.

This completes the proof.

Lemma 4.4. If $\mathcal{C}$ is a chain in $\left(E_{+}, \leq\right)$or in $\left(E_{-}, \leq\right)$, then there exists $w$ that illuminates each element of $\mathcal{C}$. 
Proof. Let $\mathcal{C}$ be a chain in $\left(E_{+}, \leq\right)$or in $\left(E_{-}, \leq\right)$. We call a chain $c_{1} \leq c_{2} \leq \ldots \leq c_{m}$ in $\left(E_{+}, \leq\right)$or in $\left(E_{-}, \leq\right)$maximal if it has length $n-1$. The chain $\mathcal{C}$ is contained in a maximal chain. As each coordinate permutation is an isometry of $\left(\mathbb{R}^{n-1},\|\cdot\|_{H}\right)$ and the map $x \mapsto-x$ is an isometry of $\left(\mathbb{R}^{n-1},\|\cdot\|_{H}\right)$, we may assume without loss of generality that $\mathcal{C}$ is contained in the maximal chain,

$$
\mathcal{C}^{*}:(1,0,0, \ldots, 0) \leq(1,1,0, \ldots, 0) \leq \ldots \leq(1,1, \ldots, 1,0) \leq(1,1,1, \ldots, 1) .
$$

Let $w \in \mathbb{R}^{n-1}$ be such that $w_{1}<w_{2}<\ldots<w_{n-1}<0$. Now if $x$ is the $k$-th element in the maximal chain and $k<n-1$, then for all $\lambda>0$ sufficiently small

$$
\|x+\lambda w\|_{H}=\left(\max _{i} x_{i}+\lambda w_{i}\right) \vee 0-\left(\min _{i} x_{i}+\lambda w_{i}\right) \wedge 0=1+\lambda w_{k}-\lambda w_{k+1}<1 .
$$

On the other hand, if $x=(1,1, \ldots, 1)$, then clearly $\|x+\lambda w\|_{H}=1+\lambda w_{n-1}<1$ for all $\lambda>0$ small. Thus $w$ illuminates each element of $\mathcal{C}^{*}$ and we are done.

To proceed we need to recall a few classical results in the combinatorics of finite partially ordered sets, see [6, Sections 9.1 and 9.2]. Firstly, we recall Dilworth's Theorem, which says that if the maximum size of an antichain in a finite partially ordered set $(P, \preceq)$ is $r$, then $P$ can be partitioned into $r$ disjoint chains. In the case where the partially ordered set is $\left(\{0,1\}^{d}, \leq\right)$, one can combine this result with Sperner's Theorem, which says that the maximum size of antichain in $\left(\{0,1\}^{d}, \leq\right)$ is $\left(\begin{array}{c}d \\ \lceil d / 2\rceil\end{array}\right)$. Thus, $\left(\{0,1\}^{d}, \leq\right)$ can be partitioned into $\left(\begin{array}{c}d \\ \lceil d / 2\rceil\end{array}\right)$ disjoint chains.

To obtain our result we need some more detailed information about the partitions. In particular, we need a result by De Bruijn,Tengbergen, Kruyswijk [3] concerning symmetric chains, see also [6, Theorem 9.3]. A chain $x^{1} \leq \ldots \leq x^{k}$ in $\left(\{0,1\}^{d}, \leq\right)$ is said to be symmetric if

(a) $\left(\sum_{j=1}^{d} x_{j}^{m}\right)+1=\sum_{j=1}^{d} x_{j}^{m+1}$ for all $1 \leq m<k$, i.e., $x^{m+1}$ is an immediate successor of $x^{m}$

(b) $\sum_{j=1}^{d} x_{j}^{k}=d-\sum_{j=1}^{d} x_{j}^{1}$.

Theorem 4.5 (De Bruijn,Tengbergen, Kruyswijk). The poset $\left(\{0,1\}^{d}, \leq\right)$ can be partitioned into $\left(\begin{array}{c}d \\ \lceil d / 2\rceil\end{array}\right)$ disjoint symmetric chains.

Let us now prove the main result of the paper.

Proof of Theorem 3.2. First recall that by Corollary 4.3 it suffices to show that $i\left(B_{H}\right) \leq\left(\begin{array}{c}n \\ \left\lceil\frac{n}{2}\right\rceil\end{array}\right)$, as $i\left(B_{\mathrm{v}}\right)=i\left(B_{H}\right)$. In other words, we only need to show that $\operatorname{ext}\left(B_{H}\right)$ can be illuminated by $\left(\begin{array}{c}n \\ \left\lceil\frac{n}{2}\right\rceil\end{array}\right)$ vectors.

There are two cases to consider: $n \geq 2$ even, and $n \geq 2$ odd.

Let us first consider the case where $n \geq 2$ is even. By Dilworth's Theorem and Sperner's Theorem we know that the partially ordered set $\left(\{0,1\}^{n-1}, \leq\right)$ can be partitioned into $\left(\begin{array}{c}n-1 \\ \left\lceil\frac{n-1}{2}\right\rceil\end{array}\right)$ disjoint chains. This implies that each of the partially ordered sets $\left(E_{+}, \leq\right)$and $\left(E_{-}, \leq\right)$can be partitioned into $\left(\begin{array}{c}n-1 \\ \left\lceil\frac{n-1}{2}\right\rceil\end{array}\right)$ disjoint chains. It now follows from Lemma 4.4 that we need at most

$$
\left(\begin{array}{c}
n-1 \\
\left\lceil\frac{n-1}{2}\right\rceil
\end{array}\right)+\left(\begin{array}{c}
n-1 \\
\left\lceil\frac{n-1}{2}\right\rceil
\end{array}\right)=\left(\begin{array}{c}
n-1 \\
\left\lfloor\frac{n-1}{2}\right\rfloor
\end{array}\right)+\left(\begin{array}{c}
n-1 \\
\left\lceil\frac{n-1}{2}\right\rceil
\end{array}\right)=\left(\begin{array}{c}
n \\
\frac{n}{2}
\end{array}\right)
$$

distinct vectors to illuminate $\operatorname{ext}\left(B_{H}\right)$. This implies that $i\left(B_{\mathrm{v}}\right)=i\left(B_{H}\right) \leq\left(\begin{array}{c}n \\ \frac{n}{2}\end{array}\right)$.

Now suppose that $n \geq 2$ is odd. By Theorem 4.5 we know that $\left(\{0,1\}^{n-1}, \leq\right)$ can be partitioned into $\left(\begin{array}{c}n-1 \\ \frac{n-1}{2}\end{array}\right)$ disjoint symmetric chains. 
Let us consider such a symmetric chain decomposition, and let

$$
\mathcal{A}_{k}:=\left\{x \in\{0,1\}^{n-1}: \sum_{i} x_{i}=k\right\},
$$

which is an antichain of size $\left(\begin{array}{c}n-1 \\ k\end{array}\right)$. Each element of $\mathcal{A}_{(n+1) / 2}$ is contained in a distinct symmetric chain, and each of these chain contains an $x \in\{0,1\}^{n-1}$ with $\sum_{i} x_{i}=(n-1) / 2$. Thus, the symmetric chain decomposition of $\left(\{0,1\}^{n-1}, \leq\right)$ consists of

$$
\left(\begin{array}{c}
n-1 \\
\frac{n+1}{2}
\end{array}\right)
$$

chains containing a vector $x$ with $\sum_{i} x_{i}=(n+1) / 2$, and

$$
\left(\begin{array}{c}
n-1 \\
\frac{n-1}{2}
\end{array}\right)-\left(\begin{array}{c}
n-1 \\
\frac{n+1}{2}
\end{array}\right)
$$

chains consisting of a single vector $x$ with $\sum_{i} x_{i}=(n-1) / 2$.

By deleting $(0,0, \ldots, 0)$ from $\{0,1\}^{n-1}$ we obtain a partition of $\left(E_{+}, \leq\right)$into disjoint chains. Let $\mathcal{S}$ be the set of vectors in $E_{+}$which form a singleton chain and $\sum_{i} x_{i}=(n-1) / 2$. So,

$$
|\mathcal{S}|=\left(\begin{array}{c}
n-1 \\
\frac{n-1}{2}
\end{array}\right)-\left(\begin{array}{c}
n-1 \\
\frac{n+1}{2}
\end{array}\right) \text {. }
$$

Now pair each $x \in E_{+}$with $x^{\prime} \in E_{-}$, where $x_{i}^{\prime}=0$ if $x_{i}=1$, and $x_{i}^{\prime}=-1$ if $x_{i}=0$. In this way we obtain a partition of $\left(E_{-}, \leq\right)$into disjoint chains with $|\mathcal{S}|$ chains consisting of a single vector. In other words, for each $x \in \mathcal{S}$ we have that $x^{\prime} \in E_{-}$forms a singleton chain in the chain decomposition of $\left(E_{-}, \leq\right)$.

We know from Lemma 4.4 that we can illuminate the $\left(\begin{array}{l}n-1 \\ \frac{n+1}{2}\end{array}\right)$ chains in $\left(E_{+}, \leq\right)$ containing a vector $x$ with $\sum_{i} x_{i}=(n+1) / 2$ using $\left(\begin{array}{l}n-1 \\ \frac{n+1}{2}\end{array}\right)$ vectors. Likewise, we can illuminate the corresponding $\left(\begin{array}{l}n-1 \\ \frac{n+1}{2}\end{array}\right)$ chains in $\left(E_{-}, \leq\right)$with $\left(\begin{array}{l}n-1 \\ \frac{n+1}{2}\end{array}\right)$ vectors. So, it remains to illuminate the singleton chains in $\left(E_{+}, \leq\right)$and $\left(E_{-}, \stackrel{2}{\leq}\right)$.

Note that if we can illuminate each pair $\left\{x, x^{\prime}\right\}$, with $x \in \mathcal{S}$ and $x^{\prime}$ the corresponding vector in $E_{-}$, by a single vector, then we need at most

$$
2\left(\begin{array}{c}
n-1 \\
\frac{n+1}{2}
\end{array}\right)+\left(\begin{array}{c}
n-1 \\
\frac{n-1}{2}
\end{array}\right)-\left(\begin{array}{c}
n-1 \\
\frac{n+1}{2}
\end{array}\right)=\left(\begin{array}{c}
n-1 \\
\frac{n-1}{2}
\end{array}\right)+\left(\begin{array}{c}
n-1 \\
\frac{n+1}{2}
\end{array}\right)=\left(\begin{array}{c}
n \\
\left\lceil\frac{n}{2}\right\rceil
\end{array}\right)
$$

vectors to illuminate $\operatorname{ext}\left(B_{H}\right)$, and hence $i\left(B_{\mathrm{v}}\right)=i\left(B_{H}\right) \leq\left(\begin{array}{c}n \\ \left\lceil\frac{n}{2}\right\rceil\end{array}\right)$ if $n \geq 2$ is odd.

To see how this can be done we consider such a pair $\left\{x, x^{\prime}\right\}$ with $x \in \mathcal{S}$ and let $I:=\left\{i: x_{i}=1\right\}$ and $J:=\left\{i: x_{i}=0\right\}$. So, $I=\left\{i: x_{i}^{\prime}=0\right\}$ and $J=\left\{i: x_{i}^{\prime}=-1\right\}$. Now let $w \in \mathbb{R}^{n-1}$ be such that $w_{i}<0$ for all $i \in I$ and $w_{i}>0$ for all $i \in J$. Then for all $\lambda>0$ sufficiently small,

$$
\|x+\lambda w\|_{H}=\max _{i \in I}\left(1+\lambda w_{i}\right)-0<1
$$

and

$$
\left\|x^{\prime}+\lambda w\right\|_{H}=0-\min _{i \in J}\left(-1+\lambda w_{i}\right)<1
$$

This shows that $w$ illuminates $x$ and $x^{\prime}$, which completes the proof. 


\section{REFERENCES}

[1] T. Bewley and E. Kohlberg, The asymptotic theory of stochastic games. Math. Oper. Res. 1(3), (1976), 197-208.

[2] V. Boltyanski, H. Martini and P.S. Soltan, Excursions into combinatorial geometry. Universitext. Springer-Verlag, Berlin, 1997.

[3] N.G. de Bruijn, C.A. van Ebbenhorst Tengbergen and D. Kruyswijk, On the set of divisors of a number. Nieuw Arch. Wiskunde (2) 23, (1951), 191-193.

[4] R. Cavazos-Cadena, Equivalence of communication and projective boundedness properties for monotone and homogeneous functions. Nonlinear Anal. 75(2), (2012), 775-785.

[5] S. Gaubert and J. Gunawardena, The Perron-Frobenius theorem for homogeneous, monotone functions. Trans. Amer. Math. Soc. 356(12), (2004), 4931-4950.

[6] S. Jukna, Extremal combinatorics. With applications in computer science. Texts in Theoretical Computer Science. An EATCS Series. Springer-Verlag, Berlin, 2001.

[7] B. Lemmens, B. Lins and R. Nussbaum, Detecting fixed points of nonexpansive maps by illuminating the unit ball, Israel J. Math., to appear, arXiv:1607.01602.

[8] B. Lemmens and R. Nussbaum, Nonlinear Perron-Frobenius theory. Cambridge Tracts in Mathematics 189, Cambridge Univ. Press, Cambridge, 2012.

[9] R.D. Nussbaum, Hilbert's projective metric and iterated nonlinear maps. Mem. Amer. Math. Soc. 75, (1988).

[10] R. D. Nussbaum. Iterated nonlinear maps and Hilbert's projective metric II. Mem. Amer. Math. Soc. 79, (1989).

[11] R. D. Nussbaum, Finsler structures for the part metric and Hilbert's projective metric and applications to ordinary differential equations, Differential Integral Equations 7(5-6), (1994), $1649-1707$.

[12] D. Rosenberg and S. Sorin, An operator approach to zero-sum repeated games. Israel J. Math. 121, (2001), 221-246.

[13] R. Schoen, The two-sex multiethnic stable population model. Theoret. Population Biol. 29(3), (1986), 343-364.

School of Mathematics, Statistics \& Actuarial Science, Sibson Building, University of Kent, Canterbury, Kent CT2 7FS, UK

E-mail address: B.Lemmens@kent.ac.uk

School of Mathematics, Statistics \& Actuarial Science, Sibson Building, University of Kent, Canterbury, Kent CT2 7FS, UK

E-mail address: 1cw32@kent.ac.uk 